\title{
Individual differences in the expression of conditioned fear are associated with endogenous fibroblast growth factor 2
}

\author{
Bronwyn M. Graham and Rick Richardson \\ School of Psychology, The University of New South Wales Australia, Sydney, 2052 New South Wales, Australia
}

\begin{abstract}
These experiments examined the relationship between the neurotrophic factor fibroblast growth factor 2 (FGF2) and individual differences in the expression of conditioned fear. Experiments 1 and 2 demonstrated that rats naturally expressing low levels of contextual or cued fear have higher levels of hippocampal FGF2 relative to rats that express high levels of conditioned fear and nonconditioned rats. Experiment 3 demonstrated that hippocampal FGF2 is not increased in rats that exhibit pharmacological-induced amnesia of conditioned fear. Together, these experiments provide evidence that FGF2 may be an endogenous regulator of fear responses to conditioned stimuli.
\end{abstract}

Only a small subset of people who experience trauma develop anxiety or trauma- and stressor-related disorders, such as posttraumatic stress disorder (PTSD) (Yehuda and LeDoux 2007). Identifying the factors associated with variations in response to adversity could aid the development of early interventions designed to augment resilience in vulnerable individuals. One approach to this issue has been to examine individual differences in trait anxiety in rodents, by measuring fear responses during anxiety-provoking situations such as novel environments, or bright and open spaces (Cryan and Holmes 2005; Graham et al. 2011). In these procedures, rats with high trait anxiety exhibit greater levels of avoidance and reduced exploration relative to rats with low trait anxiety. Recent investigations have explored the neurobiological correlates of trait anxiety in rodents. For example, Perez et al. (2009) selectively bred rats to exhibit high or low trait anxiety, and demonstrated that these phenotypic differences correlated with the neurotrophic factor fibroblast growth factor 2 (FGF2), where low anxiety rats had significantly greater hippocampal FGF2 gene expression than high anxiety rats. They further demonstrated that environmental enrichment decreased trait anxiety in highly anxious rats, an effect that was associated with an increase in hippocampal FGF2 gene expression. A reduction in trait anxiety in highly anxious rats was also achieved via chronic administration of FGF2 during adulthood (Perez et al. 2009), or a single FGF2 injection on the first day of life (Turner et al. 2011). The same correlation between trait anxiety and hippocampal FGF2 gene expression was later observed in outbred rats, in whom selective knockdown of hippocampal FGF2 activity increased anxiety (Eren-Koçak et al. 2011). Together, these findings suggest that FGF2 may be an endogenous regulator of trait anxiety, as well as a novel pharmacological anxiolytic (Graham and Richardson 2015).

The type of fear responses measured in research on trait anxiety does not require the rodent to have had any prior aversive experience, and so is considered "unlearned." In contrast, the expression of "learned fear" is contingent upon a prior aversive experience. Learned fear is commonly examined via Pavlovian fear conditioning, in which a neutral conditioned stimulus (CS, such as an auditory stimulus) is paired with an aversive uncondi-

\section{Corresponding author: bgraham@psy.unsw.edu.au}

Article is online at http://www.learnmem.org/cgi/doi/10.1101/lm.039644. 115 . tioned stimulus (US, such as a footshock), until the CS alone elicits fear responses. Fear conditioning processes are directly relevant to the etiology of trauma and stressor-related disorders, such as PTSD, which by definition develops following exposure to trauma, and involves heightened fear responses in the presence of stimuli associated with that event (Mineka and Zinbarg 2006). Just as there are large individual differences in trait anxiety, there are also individual differences in the expression of learned fear, echoing the heterogeneity of humans' responses to trauma (Bush et al. 2007; Camp et al. 2009; Duvarci et al. 2009). Exploring the neurobiological factors underlying variability in learned fear expression may prove useful in identifying specific variables that foster vulnerability or resilience following traumatic experiences.

Although a role for FGF2 in trait anxiety has been established, it is unknown whether individual differences in learned fear are similarly associated with FGF2. However, we have previously demonstrated that systemic administration of FGF2 reduces fear expression in the presence of a feared CS (Graham and Richardson 2009), similar to its anxiolytic effects during innately fear-provoking situations (Perez et al. 2009). Therefore, it is possible that rats with naturally lower levels of learned fear expression may have greater endogenous levels of FGF2, similar to what has been reported in rats with naturally low trait anxiety (Perez et al. 2009). In the present study, we assessed this possibility by examining FGF2 protein levels in several neural structures following the expression of learned fear induced by a mild conditioning event, involving a single, low-intensity footshock. In all experiments, the percentage of time spent freezing was used as an index of fear expression. Sprague-Dawley male rats, aged 8-10 wk and maintained on a $12 \mathrm{~h}$ light-dark cycle (lights on at 0700), were sacrificed $2 \mathrm{~h}$ after behavioral testing and brains were rapidly removed, snap frozen, and punch dissected for analysis of FGF2 using Western Blotting. Tissue was homogenized in lysis buffer containing protease inhibitors and total protein was quantified using a Bradford Assay. Equal amounts of protein were separated by electrophoresis on $10 \%-20 \%$ tricine SDS-polyacrylamide

\footnotetext{
(C) 2015 Graham and Richardson This article is distributed exclusively by Cold Spring Harbor Laboratory Press for the first 12 months after the full-issue publication date (see http://learnmem.cshlp.org/site/misc/terms.xhtml). After 12 months, it is available under a Creative Commons License (AttributionNonCommercial 4.0 International), as described at http://creativecommons. org/licenses/by-nc/4.0/.
} 

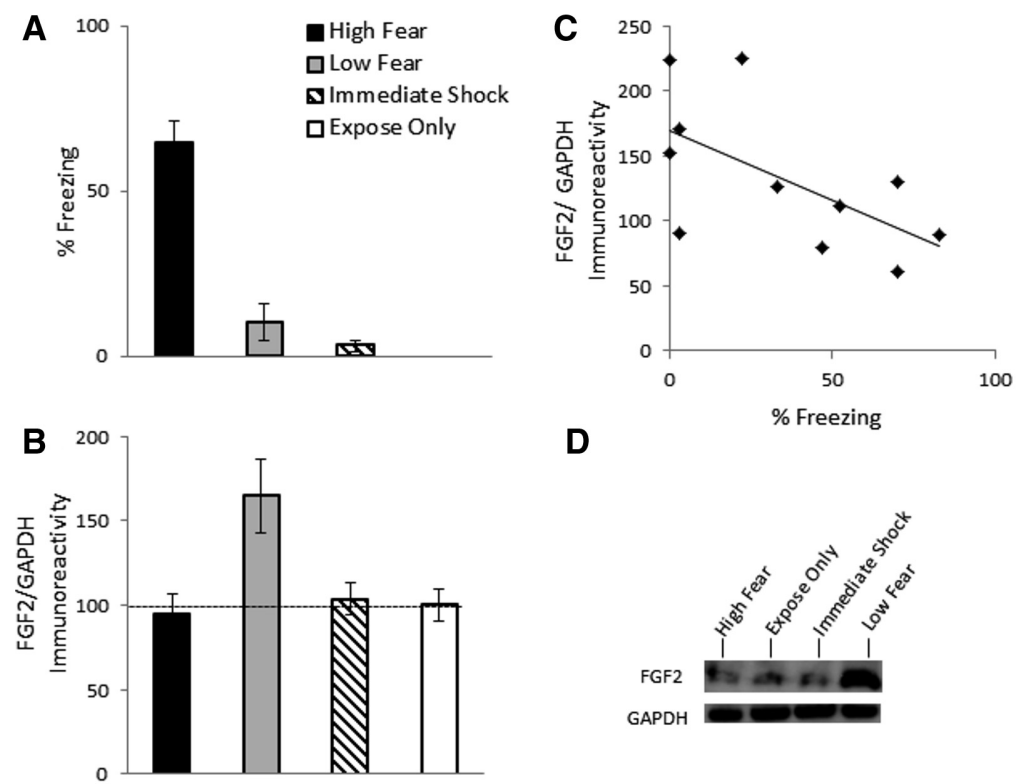

D

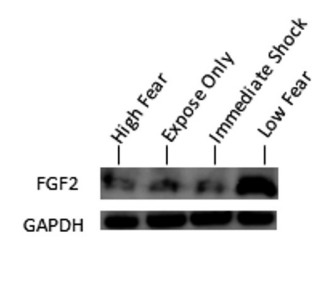

Figure 1. (A) Mean ( \pm SEM) percent freezing during test for contextual fear in Experiment 1. Rats were in one of four groups: High fear $(n=5)$, Low fear $(n=6)$, imm Shock $(n=8)$, expose only $(n=8)$. (B) Mean ( \pm SEM) FGF2/GAPDH immunoreactivity in Experiment 1. Data are presented as a percentage of mean data from group expose only. (C) Correlation between FGF2/GAPDH immunoreactivity and percent freezing for Experiment 1. (D) Representative blots for each group.

gels. Proteins were transferred to nitrocellulose membranes, and nonspecific immunoreactivity was blocked with 5\% nonfat dry milk/3\% BSA in TBST for $60 \mathrm{~min}$. Membranes were incubated overnight at $4^{\circ} \mathrm{C}$ with anti-FGF2 antibody (1:500; Abcam) or anti-GAPDH antibody (1:500; Millipore). After incubation in secondary antibody (HRP-conjugated goat anti-rabbit/anti-mouse IgG; BioRad) visualization was conducted using the ECL detection method in an ImageQuant LAS 500. The intensity of each FGF2 protein band was normalized to that of the corresponding GAPDH band, and an integrated density value was calculated using NIH Image J software. Data were analyzed using oneway Analysis of Variance and post hoc tests were conducted using StudentNewman-Keuls.

Experiment 1 assessed the relationship between FGF2 and the expression of contextual fear. On Day 1, rats were exposed to a novel context for $2 \mathrm{~min}$ prior to receiving a single $1-\mathrm{sec}, 0.6-\mathrm{mA}$ footshock. On Day 2, they were placed in the same context for $3 \mathrm{~min}$ and tested for contextual fear. Rats were divided into two groups on the basis of a median split in freezing at test (High fear and Low fear). Two additional control groups were included in this experiment: one that received an equivalent amount of context exposure on Day 1, without shock (group "expose only"), and one that received an equivalent footshock immediately upon being placed in the novel context, after which they were immediately removed (group "imm shock"). Previous research has demonstrated that immediate shock in a novel context produces negligible fear responses at test, due to the inability to form a mental representation of the context, which prevents a learned association between the context and the shock (Blanchard et al. 1976). Freezing levels on Day 2 are shown in Figure 1A. There were group differences $\left(F_{(3,26)}=58.57\right.$, $P<0.0001$ ), with High fear rats exhibiting significantly greater freezing than all other groups $(P<0.05)$, and no differences between Low fear rats and the two control groups $(P=0.14)$. There were group differences in hippocampal FGF2 expression $\left(F_{(3,26)}=4.12, P=0.018\right.$; Fig. $1 \mathrm{~B})$, with Low fear rats exhibiting significantly greater hippocampal FGF2 relative to High fear rats, as well as both control groups $(P<0.05)$. There were no differences between High fear rats and the two control groups $(P=0.9)$. In conditioned rats (i.e., Low and High fear rats), there was a significant negative correlation between freezing and hippocampal FGF2 $(r=-0.61, P=0.047$; Fig. $1 \mathrm{C})$. This correlation was no longer significant when nonconditioned rats (i.e., the two control groups) were included $(r=-0.12, P=0.53)$. The medial prefrontal cortex and amygdala were also examined for potential differences in FGF2 expression; however there were no group differences in either region ( $P s>0.83$, data not shown).

Experiment 2 assessed whether the relationship between hippocampal FGF2 levels are also associated with learned fear expression in the presence of a previously conditioned discrete cue. In
A
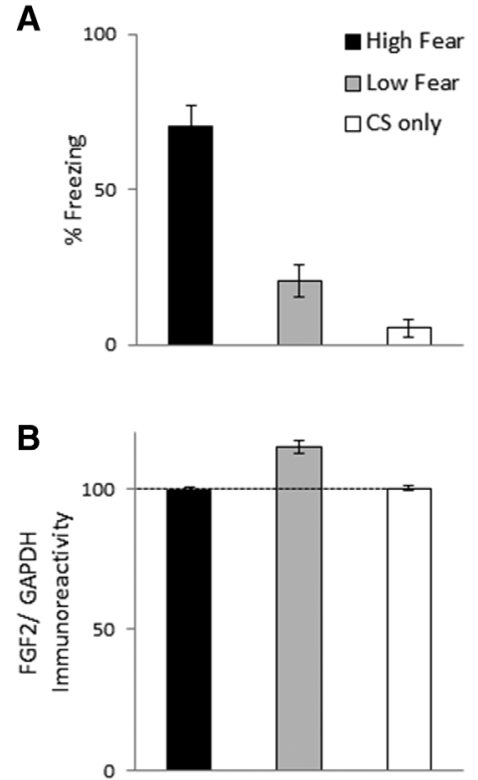

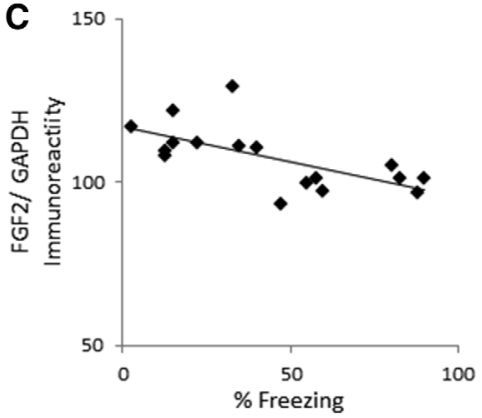

D

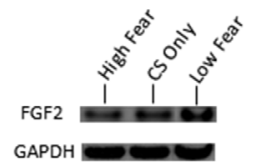

Figure 2. ( $A$ ) Mean $( \pm$ SEM) percent CS-elicited freezing in Experiment 2. Rats were in one of three groups: High fear $(n=8)$, Low fear $(n=9)$, CS only $(n=5)$. (B) Mean ( \pm SEM) FGF2/GAPDH immunoreactivity in Experiment 2. Data are presented as a percentage of mean data from group CS only. (C) Correlation between FGF2/GAPDH immunoreactivity and percent freezing for Experiment 2. (D) Representative blots for each group. 
this experiment, rats were placed in a novel context, and after 2 min, were exposed to a single 10-sec white-noise CS, which coterminated with a single 1-sec, 0.6-mA footshock. Another group of rats received the CS in the absence of the footshock (group "CS only"). All rats were tested for CS-elicited freezing in a different context the following day, in which the CS was presented for 2 min following a 1-min adaptation period. The conditioned rats were then divided into groups "High fear" and "Low fear" as in Experiment 1. While there were no group differences in pre-CS freezing during the adaptation period $\left(F_{(2,21)}=0.52, P=0.6\right.$; data not shown), groups differed in CS-elicited freezing $\left(F_{(2,21)}=45.17, \quad P<0.0001 ; \quad\right.$ Fig. $\left.2 \mathrm{~A}\right)$. High fear rats exhibited significantly greater freezing than Low fear and CS only groups, and Low fear rats exhibited slightly, but significantly, greater freezing than group CS only $(P s<0.05)$. There were group differences in hippocampal FGF2 $\left(F_{(2,21)}=23.25, \quad P<0.0001 ;\right.$ Fig. $2 \mathrm{~B})$, with Low fear rats exhibiting significantly greater hippocampal FGF2 relative to High fear rats, as well group CS only $(P<0.05)$; the latter two groups did not differ $(P=0.83)$. In conditioned rats (i.e., High and Low fear groups), there was a significant negative correlation between freezing and hippocampal FGF2 $(r=-0.66, P=0.004$; Fig. 2C). This correlation was no longer significant when rats from group CS only were included $(r=-0.31, P=0.16)$. The amygdala was also examined for potential differences in FGF2 expression, however there were no group differences $\left(F_{(2,21)}=1.67, P=0.21\right.$, data not shown).

In Experiments 1 and 2, the low level of freezing in Low fear rats may reflect a failure in the acquisition or consolidation of the association between the context/CS and the US. Thus, it is possible that higher levels of FGF2 are indicative of poor associative memory, as opposed to greater resilience, following fear conditioning. If so, then rats that have been subjected to a manipulation that produces amnesia for conditioned fear should also exhibit increased FGF2 expression. In Experiment 3, this possibility was assessed by giving rats a subcutaneous injection of the NMDA receptor antagonist MK801 $(0.1 \mathrm{mg} / \mathrm{kg})$ prior to contextual fear conditioning as in Experiment 1. Rats treated with MK801 prior to fear conditioning typically exhibit low fear at test due to a failure to acquire and/or consolidate the fear memory (Graham and Richardson 2011a). Another group of rats received a vehicle injection prior to contextual fear conditioning, and were divided into High and Low fear groups following test for contextual fear on Day 2 as in the previous experiments. There were group differences in freezing during test $\left(F_{(2,37)}=97.39, P<0.0001\right.$; Fig. 3A). High fear rats exhibited significantly greater freezing than Low fear and MK801 groups, and MK801-treated rats showed slightly, but significantly, lower freezing than Low fear rats $(P \mathrm{~s}<0.05) .{ }^{1}$

\footnotetext{
${ }^{1}$ An additional control group $(n=8)$ that received MK801 prior to both training and test was included (mean freezing at test $=10.75 \%$, SEM $=7.3$ ), which confirmed that the freezing deficit observed in MK801-treated rats was not due to state-dependent effects (no difference in context-elicited freezing compared with group MK801; $P=0.29$ ).
}

There were group differences in hippocampal FGF2 $\left(F_{(2,37)}=\right.$ $9.52, P=0.001)$, with Low fear rats exhibiting significantly greater hippocampal FGF2 relative to High fear rats as well as MK801-treated rats $(P<0.05)$. There were no differences in hippocampal FGF2 between High fear and MK801-treated rats $(P=$ 0.31). In vehicle-treated rats (i.e., High and Low fear groups), there was a significant negative correlation between freezing and hippocampal FGF2 $(r=-0.645, P=0.001)$. This correlation reduced substantially, but remained significant, when MK801-treated rats were included $(r=-0.398, P=0.013)$.

Together, these experiments demonstrate that rats with naturally low contextual or cued conditioned fear expression exhibit higher hippocampal FGF2 relative to rats with naturally high conditioned fear expression. Hippocampal FGF2 does not appear to be negatively associated with mere freezing behavior, because Low fear rats had higher FGF2 relative to (1) rats that merely received exposure to the context without shock (Experiment 1), (2) rats that received an immediate shock with minimal context exposure (and thus exhibit negligible fear responses at test; Experiment 1), and (3) rats that were presented the CS without shock (Experiment 2). All these groups exhibited similar, or even lower, freezing relative to Low fear conditioned rats, yet their FGF2 was comparable to that of High fear rats. Furthermore, greater FGF2 in Low fear rats does not appear to reflect a failure to learn or consolidate the conditioned association, because rats that were given MK801 prior to contextual fear conditioning (which disrupts the consolidation of learned fear) had comparable FGF2 expression to High fear rats, although they exhibited even lower freezing than Low fear rats. A relationship between FGF2 and learned fear expression, as opposed to mere freezing behavior, is also supported by our three correlational analyses, which demonstrated a consistent significant negative correlation between FGF2 and freezing only in rats that received the conditioning procedure (in the absence of MK801).

We have previously demonstrated that FGF2 facilitates the consolidation of fear extinction, which is the reduction in learned 
fear that occurs following repeated presentations of a CS in the absence of the US (Graham and Richardson 2009, 2010, 2011b). Therefore, it is possible that Low fear rats, facilitated by high FGF2, exhibited very rapid extinction during test. However, we view this possibility as unlikely for several reasons. First, while systemic FGF2 reduces overall freezing during extinction, it does not increase the rate of extinction, but rather, enhances its long-term consolidation, which is evident by reduced freezing in the presence of the extinguished CS $24 \mathrm{~h}$ after extinction training (Graham and Richardson 2009). Second, FGF2 facilitates extinction when infused directly into the amygdala (Graham and Richardson 2011b), and yet our results show a relationship between learned fear expression and hippocampal FGF2, but not the amygdala. Third, an analysis of freezing in High and Low fear rats across the 3-min test revealed that both groups exhibited low freezing initially, which was maintained in Low fear rats, whereas freezing in High fear rats increased across test (i.e., as opposed to freezing in Low fear rats decreasing more quickly across test, as would occur with rapid extinction; data not shown).

Rather than facilitating rapid extinction, our findings suggest that FGF2 may be an endogenous regulator of emotional responses to previously conditioned contexts and cues. It may be considered surprising that differential FGF2 expression was observed in the hippocampus, and not the amygdala, even in rats that had undergone cued conditioning (Experiment 2). However, it is possible that the individual differences in cued and contextual fear expression observed in the present experiments reflect differences in "conditionability" more generally (as opposed to differential associative learning), which could be driven by broader trait-like differences in anxiety propensity. This interpretation is consistent with previous findings (e.g., Perez et al. 2009, Turner et al. 2011) indicating that hippocampal FGF2 is negatively associated with trait anxiety. The increase in FGF2 in Low fear conditioned rats may reflect an innate ability to inhibit fear in the presence of conditioned contexts and cues. Such a role for endogenous FGF2 is consistent with our previous finding that systemic administration of FGF2 leads to reduced CS-elicited freezing (Graham and Richardson 2009). Future studies in which the impact of blocking FGF2 activity using a neutralizing antibody (e.g., Hafenbreidel et al. 2015) are required in order to determine FGF2's causative role in learned fear expression. Nevertheless, these preliminary findings raise the intriguing possibility that the ability to recruit FGF2 in the presence of conditioned feared contexts and stimuli may in part underlie risk or resilience in the development of pathological anxious responses following traumatic experiences.

\section{Acknowledgment}

This work was supported by a National Health and Medical Research Council project grant (APP1031688) to R.R., and a
University of New South Wales Goldstar Award to B.M.G. and R.R. B.M.G. received salary support from an Australian Research Council Discovery Early Career Research Award (DE140100243).

\section{References}

Blanchard RJ, Fukunaga KK, Blanchard DC. 1976. Environmental control of defensive reactions to footshock. Bull Psychon Soc 8: $129-130$.

Bush DE, Sotres-Bayon F, LeDoux JE. 2007. Individual differences in fear: isolating fear reactivity and fear recovery phenotypes. J Trauma Stress 20: $413-422$.

Camp M, Norcross M, Whittle N, Feyder M, D'Hanis W, Yilmazer-Hanke D, Singewald N, Holmes A. 2009. Impaired Pavlovian fear extinction is a common phenotype across genetic lineages of the 129 inbred mouse strain. Genes Brain Behav 8: 744-752.

Cryan JF, Holmes A. 2005. The ascent of mouse: advances in modelling human depression and anxiety. Nat Rev Drug Discov 4: 775-790.

Duvarci S, Bauer EP, Paré D. 2009. The bed nucleus of the stria terminalis mediates inter-individual variations in anxiety and fear. J Neurosci 29: 10357-10361.

Eren-Koçak E, Turner CA, Watson SJ, Akil H. 2011. Short-hairpin RNA silencing of endogenous fibroblast growth factor 2 in rat hippocampus increases anxiety behavior. Biol Psychiatry 69: 534-540.

Graham BM, Richardson R. 2009. Acute systemic fibroblast growth factor-2 enhances long-term extinction of fear and reduces reinstatement in rats. Neuropsychopharmacology 34: 1875-1882.

Graham BM, Richardson R. 2010. Fibroblast growth factor-2 enhances extinction and reduces renewal of conditioned fear. Neuropsychopharmacology 35: 1348-1355.

Graham BM, Richardson R. 2011a. Fibroblast growth factor-2 alters the nature of extinction. Learn Mem 18: $80-84$.

Graham BM, Richardson R. 2011b. Intraamygdala infusion of Fibroblast Growth Factor 2 enhances extinction and reduces renewal and reinstatement in adult rats. J Neurosci 31: 14151-14157.

Graham BM, Richardson R. 2015. Fibroblast growth factor 2 as a new approach to fighting fear. JAMA Psychiatry 72: 959-960.

Graham BM, Langton JM, Richardson R. 2011. Pharmacological enhancement of fear reduction: preclinical models. Br J Pharmacol 164: 1230-1247.

Hafenbreidel M, Twining RC, Todd CR, Mueller D. 2015. Blocking infralimbic basic fibroblast growth factor (bFGF or FGF2) facilitates extinction of drug seeking after cocaine self-administration. Neuropsychopharmacology. doi: 10.1038/npp.2015.144

Mineka S, Zinbarg R. 2006. A contemporary learning theory perspective on the etiology of anxiety disorders: it's not what you thought it was. Am Psychol 61: 10-26.

Perez JA, Clinton SM, Turner CA, Watson SJ, Akil H. 2009. A new role for FGF2 as an endogenous inhibitor of anxiety. J Neurosci 29: 6379-6387.

Turner CA, Clinton SM, Thompson RC, Watson SJ Jr, Akil H. 2011. Fibroblast growth factor-2 (FGF2) augmentation early in life alters hippocampal development and rescues the anxiety phenotype in vulnerable animals. Proc Natl Acad Sci 108: 8021-8025.

Yehuda R, LeDoux J. 2007. Response variation following trauma: a translational neuroscience approach to understanding PTSD. Neuron 56: $19-32$.

Received July 27, 2015; accepted in revised form October 13, 2015. 


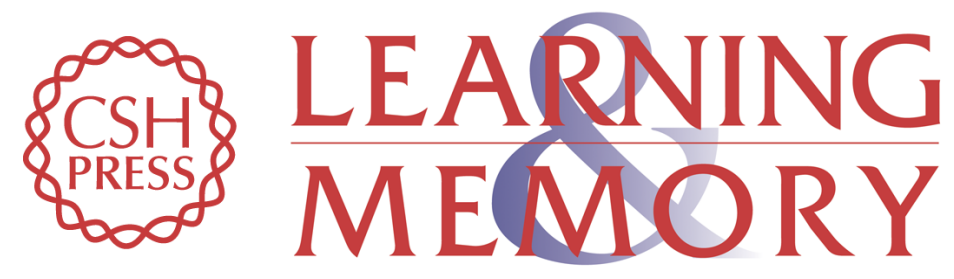

\section{Individual differences in the expression of conditioned fear are associated with endogenous fibroblast growth factor 2}

Bronwyn M. Graham and Rick Richardson

Learn. Mem. 2016, 23:

Access the most recent version at doi:10.1101/Im.039644.115

References This article cites 17 articles, 5 of which can be accessed free at: http://learnmem.cshlp.org/content/23/1/42.full.htmI\#ref-list-1

Creative This article is distributed exclusively by Cold Spring Harbor Laboratory Press for the Commons License first 12 months after the full-issue publication date (see

http://learnmem.cshlp.org/site/misc/terms.xhtml). After 12 months, it is available under a Creative Commons License (Attribution-NonCommercial 4.0 International), as described at http://creativecommons.org/licenses/by-nc/4.0/.

Email Alerting Receive free email alerts when new articles cite this article - sign up in the box at the Service top right corner of the article or click here. 